\title{
Laparoscopic ventral mesh rectopexy versus laparoscopic ventral mesh rectopexy combined with trans-anal rectal resection in the treatment of obstructed defecation syndrome: study protocol for a randomized controlled trial
}

Yuhang Ge

Wuhan University Renmin Hospital

Chuntao Wang

Wuhan University Renmin Hospital

\section{Peng Wang}

Wuhan University Renmin Hospital

Xu Zhu

Wuhan University Renmin Hospital

Wenyi Guo

Wuhan University Renmin Hospital

Tao Fu ( $\sim$ futao1975@tom.com )

Wuhan University Renmin Hospital https://orcid.org/0000-0003-4713-7269

Study protocol

Keywords: obstructed defecation syndrome, laparoscopic ventral mesh rectopexy, stapled trans-anal rectal resection, constipation, rectocele, enterocele, intussusception

Posted Date: March 22nd, 2019

DOI: https://doi.org/10.21203/rs.2.474/v1

License: (c) (1) This work is licensed under a Creative Commons Attribution 4.0 International License.

Read Full License 


\section{Abstract}

Background: Laparoscopic ventral mesh rectopexy (LVMR) and stapled trans-anal rectal resection (STARR) are effective and well-recognized treatments for symptoms of obstructed defecation syndrome (ODS) in the context of rectal prolapse and rectocele. However, recurrence and postoperative complications including symptomatic grade III/IV hemorrhoids develop in patients undergo LVMR. It was hypothesized that adding STARR to LVMR is effective in improving the clinical symptoms and functional outcomes and reducing the postoperative complications after repair of ODS. Methods/Design: In this prospective, single-blind, randomised trial, consecutive patients aged 18 years or older at a single center in China with ODS are randomly assigned (1:1) to either LVMR or LVMR plus STARR. Functional assessment is done preoperatively and 12 months postoperatively. The primary outcome is the rate of complications started in the hospital and is followed up in the outpatient setting until 12 months after surgery. Patients and those assessing the outcomes were masked to the procedure. The primary analysis is done in the per-protocol population. Outcomes are assessed in the entire cohort. Discussion: This study will add to the scant literature regarding surgical management of ODS.

\section{Background}

Obstructed defecation syndrome (ODS) is characterized by a prolonged history of difficult or inadequate rectal evacuation, including excessive straining, feeling of incomplete evacuation, sensation of obstruction during defecation, passage of hard stools, and/or inability to evacuate without inserting a finger into the vagina or anus to evacuate bowel contents [1]. It is found in approximately half of patients with chronic constipation and typically seen in middle-aged, multiparous women [2-4]. Prevalence ranges from $3.4 \%$ in the general population up to $23 \%$ in middle-aged women [5]. ODS may result from inadequate rectal propulsive forces and/or increased resistance to evacuation and can be related to many anatomic and functional abnormalities. The determinants of ODS have long been debated and not very well understood. Rectocele, enterocele, and intussusception have been identified as anatomic abnormalities [6], and anxiety, depression, dyssynergia, and pudendal neuropathy as functional disorders that contribute to ODS [7].

In most international practices, patients with ODS are offered first-line conservative treatment such as lifestyle advice and laxatives [8]. Then referred to nurse-led bowel retraining programmes with a variety of behavioral interventions including biofeedback and psychosocial support to improve defecatory function [9]. Patients failing behavioral interventions may progress to transanal irrigation [10]. However despite those approaches, more than half of the patients will have persistent intractable symptoms [11].

When conservative interventions fail, surgical treatment is usually offered. More than 100 surgical procedures have been described in the literature [12]. These procedures can be divided into perineal and abdominal techniques. The optimal surgical management of ODS is still under debate with many procedures associated with high rates of recurrence. The PROSPER trial reported that 3-year recurrence rates were about $24 \%$ for Altemeier's procedure and $31 \%$ for Deloreme's procedure $[13,14]$. A meta- 
analysis involving 39 studies with 2647 patients showed that the median incidences of recurrence were $11.4 \%$ for Altemeier's procedure, $14.4 \%$ for Deloreme's procedure, and $13.9 \%$ for perineal stapled prolapse resection [15]. The laparoscopic ventral mesh rectopexy (LVMR) procedure attaches the rectum to an approximately normal anatomical position and has been shown to improve functional and quality of life outcomes in patients with incontinence and obstructive defecation [16, 17]. Available data suggests that it is associated with a low recurrence rate of $3 \%-5 \%$ [18-20].

However, LVMR has been shown to be associated with various complications. High-grade hemorrhoids is one of them and always requiring surgery [21]. It was hypothesized that adding stapled trans-anal rectal resection (STARR) to LVMR is effective in improving the clinical symptoms and functional outcomes and reducing the postoperative complications after repair of ODS. In order to prove this hypothesis, we compare LVMR alone with LVMR combined with STARR to evaluated the role of STARR in improving the clinical symptoms after repair of ODS. This trial studies how well LVMR with or without STARR works in treating patients with ODS.

\section{Methods/design}

This trial is carried out in accordance with the Declaration of Helsinki and Good Clinical Practice Guidelines. It has been approved by the Ethics Committee at Renmin Hospital of Wuhan University (approval number WDRY2017-K008). A patient information sheet will be given to patients who are willing to consider trial entry, and a written informed consent is obtained before they are included. The LVMRSTARRODS2017 trial has been registered at ClinicalTrials.gov [NCT03060330].

\section{Study design}

A single center, prospective, randomized controlled, parallel, and single-blind trial was designed. The LVMRSTARRODS2017 trial compares LVMR with or without STARR in women undergoing surgery for ODS. The overall design is shown in Fig. 1.

\section{Study objectives}

The aim of the study is to determine if LVMR combined with STARR helps patients with ODS more than LVMR alone. Patients are blinded and are not aware undergoing which group of procedures. For the surgeon performs the procedures and will see patients postoperatively, double blinding could not be guaranteed. However, with an independent masked evaluator performing the clinical assessment, the bias of a single-blind study will be corrected.

\section{Primary endpoint}

The primary study endpoint is defined as the rate of complications within 12 months after surgery.

\section{Secondary endpoints}


The secondary endpoints are defined as the percentage of changes in total ODS score (ODS-S), Patient Assessment of Constipation-Quality of Life score (PAC-QoL), the Health-Related Quality of Life Short Form Health Survey (SF-36) version 1, the Cleveland Clinic Fecal Incontinence Score (CCFI), the Fecal Incontinence Quality of Life Scale (FIQoL), between baseline and 1, 3, 6 and 12 months follow-up.

\section{Eligibility criteria}

Eligible patients are adults aged 18 years or older, no more than 70 . The eligibility criteria are listed in Table 1.

Table 1 Eligibility criteria.

Inclusion criteria

- Patients affected by obstructed defecation with a minimum ODS score of 10

- Have rectocele and/or rectal prolapse confirmed by defecography

- Often experience excessive straining, sense of incomplete evacuation, and/or prolonged time for complete evacuation when attempting a bowel movement

- Have experienced ODS symptoms for at least 12 months prior to enrollment

- Failure of at least 6 months of medical therapy

- Have an American Society of Anesthesiologists (ASA) score of no more than 3
Exclusion criteria

· Full-thickness prolapse

- Slow transit constipation

$\cdot$ Anismus

- Pelvic organ prolapse

- Previous rectal or anal surgery

- Recto-vaginal fistula

- Pregnancy

- Prior pelvic radiotherapy

- Significant rectal fibrosis

- Evidence of colorectal neoplasia, carcinoma, or inflammatory bowel disease

- Perineal infection

\section{Surgical intervention}

All the procedures are performed by a single surgeon (TF). The LVMR technique used for this protocol is as previously described $[18,19]$. Subjects randomized to this arm will receive a standard LVMR. Briefly, the peritoneum is opened over the right side of the sacral promontory. The mesorectum is dissected and extended in an inverted $\mathrm{J}$-form along the right side. The rectovaginal septum is opened to the pelvic floor. Polypropylene mesh is pasted to the ventral aspect of the rectum with biological glue. The proximal end of the mesh is fixed on the anterior longitudinal ligament at sacral promontory with nonabsorbable 
sutures. The incised peritoneum is then closed to over the mesh. Subjects randomized to the LVMR with STARR arm will undergo a STARR procedure after a standard LVMR. Briefly, after finishing abdominal procedures, a circular anal dilator is then introduced into the rectum. The purse-string anoscope is used to complete a purse-string suture around the entire rectal ciecumference, about 2-3 cm above the haemorrhoidal apex. A 33-mm circular stapler is opened, inserted until crossing the purse-string which is then tied with a knot. Then the stapler is closed and fired.

\section{Recruitment and trial timeline}

Enrolling study participants was started in February 2017. The duration of the study for each patient is expected to be 12 months with follow-up at 1, 3, 6 and 12 months postoperatively.

\section{Treatment assignment and randomization}

Eligible patients receive information about participating in the trial by their surgeon. Consecutive eligible patients are randomly assigned to one of two groups with a 1:1 equal allocation ratio. An independent individual performs randomization using a software randomization program.

\section{Assessments}

\section{Baseline}

After recruitment and consent, the following baseline data will be obtained within one months after surgery (Table 2): demographic information, medical history, list of medications, and physical exam. The coherent and easily administered SF-36 is used as a generic measure of health-related quality of life [23]. The PAC-QoL and FIQoL are used for patients with constipation or fecal incontinence, respectively. Functional assessment is performed for constipation using the ODS-S, for fecal incontinence using the CCIS.

\section{Data collection}

Table 2 shows the timeline of visits, events and data collection.

Table 2 Schedule of study measures. 


$\begin{array}{llllll}\text { Baseline Surgery } & 1 & 3 & 6 & 12 \\ & & \text { months } & \text { months } & \text { months } & \text { months }\end{array}$

\begin{tabular}{|c|c|c|c|c|c|}
\hline $\begin{array}{l}\text { Window for collecting } \\
\text { measure }\end{array}$ & $\begin{array}{l}-2 \\
\text { months }\end{array}$ & & & & \\
\hline Informed consent & $x$ & & & & \\
\hline Demographics & $x$ & & & & \\
\hline Medical history & $x$ & & & & \\
\hline Medications & $x$ & $x$ & $x$ & $x$ & $x$ \\
\hline Physical exam & $x$ & $x$ & $x$ & $x$ & $x$ \\
\hline ODS-S & $x$ & $x$ & $x$ & $x$ & $x$ \\
\hline PAC-QoL & $x$ & $x$ & $x$ & $x$ & $x$ \\
\hline SF-36 & $x$ & $x$ & $x$ & $x$ & $x$ \\
\hline CCFI & $x$ & $x$ & $x$ & $x$ & $x$ \\
\hline FIQoL & $x$ & $x$ & $x$ & $x$ & $x$ \\
\hline Defecography & $x$ & & & $x$ & $x$ \\
\hline
\end{tabular}

\section{Statistical analysis}

\section{Sample size}

The LVMRSTARRODS2017 trial is a superiority trial with hypothesis that the LVMR plus STARR approach reducing the primary endpoint events. The sample size was calculated with combined results from published studies on LVMR. A systematic review shows that abdominal rectopexy results in a combined recurrence and complication rate of $20.8 \%$ [22]. A previous study showed $24.3 \%$ of the patients after LVMR developed symptomatic grade III/IV hemorrhoids requiring stapled or excisional hemorrhoidectomy. Taken together, these data indicate that the prevalence of all complications including symptomatic hemorrhoids is estimated to be $45 \%$. Accordingly, it was calculated that a total of 92 patients (46 in each group) would be sufficient to detect an absolute risk difference of $15 \%$ for the primary endpoint with significance $\alpha=5 \%$, achieving a power of $1-\beta=80 \%$. With an anticipated $10 \%$ rate of dropout and loss to follow-up, a total of 102 patients, equally divided into both groups, are allocated to the trial.

\section{Statistical analysis plan}

SPSS software version 20.0 is used for statistical analyses. Data will be presented as mean \pm standard deviation, median and interquartile range, or number and percentage. Comparison of continuous variables is performed using unpaired t-test or Wilcoxon signed rank test. Chi-square test or Fisher's exact 
test according to the expected counts is applied to compare categorical variables. For adjusted analysis, linear or logistic regression modeling is used for continuous or categorical outcome measures, respectively. In all cases, $P<0.05$ is considered significant.

\section{Discussion}

This trial is designed to find out whether adding the STARR to LVMR approach will lead to an improvement of clinical symptoms and functional outcomes and a reduction of complications compared to LVMR approach along in patients with ODS.

LVMR is widely used by surgeons for the treatment of ODS in the context of rectocele, enterocele, and intussusception (including rectal prolapse) [24]. More and more evidence shows favorable long-term results in terms of function, anatomical correction and low rates of morbidity and recurrence in patients with ODS after LVMR $[20,25,26]$. However, LVMR has potential complications including functional and mesh-related complications as well. The complications include mesh erosion, infection, visceral injury, mesh failure [27-29]. Developing high-grade hemorrhoids has also been recognized as a common complication after LVMR and it may act as precursor of recurrence and always requires surgery [21]. Of course, some of those complications may be reduced when the surgeons possessing the appropriate expertise and getting trained to ensure most favorable results and minimize the risk, but some complications are related to design defects of the procedure itself.

STARR procedure was first described by Longo with the goal to remove redundant rectal wall by using a circular stapler [30]. Although several other specialized staplers have been developed which aims to resect more prolapsed tissue to improve the outcome [31-34], serious complications, including rectal perforation, severe bleeding with large hematoma formation, and sepsis, etc. have been reported to be associated with STARR procedure. Other complications, fecal incontinence, rectovaginal fistula formation and intractable pelvic pain, etc. have also been reported [35-38].

We hypothesized that combining LVMR with STARR is helpful for the treatment of ODS based on following rationales: LVMR has an advantage of sufficient exposure not only of anterior rectal wall but other pelvic organs as well. However, LVMR contains attaching a mesh anteriorly to the lower rectum and posteriorly to the sacral promontory without mobilizing rectum posteriorly, it is possible that repair of the anatomy may not be fully achieved with this procedure. STARR procedure on the other hand has an advantage of sufficient exposure of the low part of rectum and especially its posterior wall, and theoretically, it may resect the additional prolapsed tissue after LVMR. Moreover, for LVMR restores at least part of the anatomy, supplementary STARR does not need to excise too much prolapsed tissue to improve the outcome which lower the rates of serious complications.

One of the potential limitations for combined approach is that perioperative complications (e.g. perforation, bleeding with large hematoma formation and sepsis) may be more difficult to manage when compared to complications occurring during single procedure. Adding STARR to LVMR may increase the cost of hospitalization and the risk for potential complications. Furthermore, LVMR and STARR are 
advanced procedures that not only require the expertise from colorectal surgeons, but also the special involvement of interventional radiologists and gynecologists to manage potential related complications. For this reason, this trial will be performed in one single expert center with multidisciplinary expertise.

In summary, considering various treatments for ODS, conservative and surgical, which reflects the low evidence level in this field. The ideal surgical procedures for ODS remains unclear. The purpose of this prospective, randomized controlled clinical trial is to compare function, anatomy, morbidity and recurrence after LVMR combined with STARR and LVMR alone in patients with ODS. This study will make an important contribution to the literature.

\section{Trial status}

Enrolment in the trial started in April 2017. At the time of the submission of this paper (January 2019), the protocol version is version Initially the study was planned to finish in April 2020, but due to difficulties with recruitment, we have recently obtained an extension of enrolment into the study to continue into 2022. The completion date is estimated to be April 2023.

\section{Declarations}

\section{Ethics approval and consent to participate}

It has been approved by the Ethics Committee at Renmin Hospital of Wuhan University (approval number WDRY2017-K008). Patients willing to participate in the trial will be provided with a patient information sheet. They will be included after written informed consent is obtained.

\section{Consent for publication}

Not applicable.

\section{Availability of data and material}

The datasets used and/or analyzed will be available from the corresponding author on reasonable request after the current study is complete.

\section{Competing interests}

The authors declare that they have no competing interests.

\section{Funding}

This project was funded by the Bethune-Ethicon Excellence in Surgery Grant. The funder had no role in the design and conduct of the study; collection, management, analysis and interpretation of the data; preparation, review, or approval of the manuscript; and decision to submit the manuscript for publication. 
TF and YHG drafted the manuscript. TF is the principal investigator for the study. CTW, PW, and TF are responsible for the concept and design. YG, PW, and TF made significant contributions to protocol validity, design, and drafting and revision of the manuscript. XZ and WG developed the statistical considerations for the trial. All authors contributed to the scientific accuracy of the manuscript and read and approved the final manuscript.

\section{Acknowledgements}

None.

\section{Abbreviations}

ODS: obstructed defecation syndrome; LVMR: laparoscopic ventral mesh rectopexy; STARR: stapled trans-anal rectal resection; ODS-S: obstructed defecation syndrome score; PAC-QoL: Patient Assessment of Constipation-Quality of Life score; SF-36: Health-Related Quality of Life Short Form Health Survey; CCFI: Cleveland Clinic Fecal Incontinence Score; FIQoL: Fecal Incontinence Quality of Life Scale

\section{References}

[1] C.W. Hicks, M. Weinstein, M. Wakamatsu, L. Savitt, S. Pulliam, L. Bordeianou, In patients with rectoceles and obstructed defecation syndrome, surgery should be the option of last resort, Surgery 155(4) (2014) 659-67.

[2] M. Khaikin, S.D. Wexner, Treatment strategies in obstructed defecation and fecal incontinence, World J. Gastroenterol. 12(20) (2006) 3168-73.

[3] S.S. Rao, Dyssynergic defecation, Gastroenterol. Clin. North Am. 30(1) (2001) 97-114.

[4] E. Surrenti, D.M. Rath, J.H. Pemberton, M. Camilleri, Audit of constipation in a tertiary referral gastroenterology practice, Am. J. Gastroenterol. 90(9) (1995) 1471-5.

[5] W.F. Stewart, J.N. Liberman, R.S. Sandler, M.S. Woods, A. Stemhagen, E. Chee, R.B. Lipton, C.E. Farup, Epidemiology of constipation (EPOC) study in the United States: relation of clinical subtypes to sociodemographic features, Am. J. Gastroenterol. 94(12) (1999) 3530-40.

[6] P.M. Cavallaro, K. Staller, L.R. Savitt, H. Milch, K. Kennedy, M.M. Weinstein, R. Ricciardi, L.G. Bordeianou, The Contributions of Internal Intussusception, Irritable Bowel Syndrome, and Pelvic Floor Dyssynergia to Obstructed Defecation Syndrome, Dis. Colon Rectum 62(1) (2019) 56-62.

[7] M. Pescatori, M. Spyrou, A. Pulvirenti d'Urso, A prospective evaluation of occult disorders in obstructed defecation using the 'iceberg diagram', Colorectal Dis. 9(5) (2007) 452-6.

[8] M. Camilleri, A.C. Ford, G.M. Mawe, P.G. Dinning, S.S. Rao, W.D. Chey, M. Simren, A. Lembo, T.M. YoungFadok, L. Chang, Chronic constipation, Nat Rev Dis Primers 3 (2017) 17095. 
[9] S.S.C. Rao, J.A. Valestin, X. Xiang, S. Hamdy, C.S. Bradley, M.B. Zimmerman, Home-based versus office-based biofeedback therapy for constipation with dyssynergic defecation: a randomised controlled trial, Lancet Gastroenterol Hepatol 3(11) (2018) 768-777.

[10] T. Juul, P. Christensen, Prospective evaluation of transanal irrigation for fecal incontinence and constipation, Tech. Coloproctol. 21(5) (2017) 363-371.

[11] V. Podzemny, L.C. Pescatori, M. Pescatori, Management of obstructed defecation, World J. Gastroenterol. 21(4) (2015) 1053-60.

[12] S. Rothenhoefer, F. Herrle, A. Herold, A. Joos, D. Bussen, M. Kieser, P. Schiller, C. Klose, C.M. Seiler, P. Kienle, S. Post, DeloRes trial: study protocol for a randomized trial comparing two standardized surgical approaches in rectal prolapse - Delorme's procedure versus resection rectopexy, Trials 13 (2012) 155.

[13] C.K. Gunner, A. Senapati, J.M. Northover, S.R. Brown, Life after PROSPER. What do people do for external rectal prolapse?, Colorectal Dis. 18(8) (2016) 811-4.

[14] A. Senapati, R.G. Gray, L.J. Middleton, J. Harding, R.K. Hills, N.C. Armitage, L. Buckley, J.M. Northover, P.C. Group, PROSPER: a randomised comparison of surgical treatments for rectal prolapse, Colorectal Dis. 15(7) (2013) 858-68.

[15] S.H. Emile, H. Elfeki, M. Shalaby, A. Sakr, P. Sileri, S.D. Wexner, Perineal resectional procedures for the treatment of complete rectal prolapse: A systematic review of the literature, Int. J. Surg. 46 (2017) 146154.

[16] J.W. van den Esschert, A.A. van Geloven, N. Vermulst, A.G. Groenedijk, L.T. de Wit, M.F. Gerhards, Laparoscopic ventral rectopexy for obstructed defecation syndrome, Surg. Endosc. 22(12) (2008) 272832.

[17] P.H. Pucher, D. Mayo, A.R. Dixon, A. Clarke, M.J. Lamparelli, Learning curves and surgical outcomes for proctored adoption of laparoscopic ventral mesh rectopexy: cumulative sum curve analysis, Surg. Endosc. 31(3) (2017) 1421-1426.

[18] A. D'Hoore, R. Cadoni, F. Penninckx, Long-term outcome of laparoscopic ventral rectopexy for total rectal prolapse, Br. J. Surg. 91(11) (2004) 1500-5.

[19] A. D'Hoore, F. Penninckx, Laparoscopic ventral recto(colpo)pexy for rectal prolapse: surgical technique and outcome for 109 patients, Surg. Endosc. 20(12) (2006) 1919-23.

[20] J. Randall, E. Smyth, K. McCarthy, A.R. Dixon, Outcome of laparoscopic ventral mesh rectopexy for external rectal prolapse, Colorectal Dis. 16(11) (2014) 914-9.

[21] J.J. van lersel, H.A. Formijne Jonkers, P.M. Verheijen, W.A. Draaisma, E.C. Consten, I.A. Broeders, Highgrade hemorrhoids requiring surgical treatment are common after laparoscopic ventral mesh rectopexy, 
Tech. Coloproctol. 20(4) (2016) 235-42.

[22] S.H. Emile, H.A. Elfeki, M. Youssef, M. Farid, S.D. Wexner, Abdominal rectopexy for the treatment of internal rectal prolapse: a systematic review and meta-analysis, Colorectal Dis. 19(1) (2017) 013-024.

[23] A.M. Garratt, D.A. Ruta, M.I. Abdalla, J.K. Buckingham, I.T. Russell, The SF36 health survey questionnaire: an outcome measure suitable for routine use within the NHS?, BMJ 306(6890) (1993) 1440-4.

[24] K. Horisberger, A. Rickert, S. Templin, S. Post, P. Kienle, Laparoscopic ventral mesh rectopexy in complex pelvic floor disorder, Int. J. Colorectal Dis. 31(5) (2016) 991-6.

[25] R.K. Mehmood, J. Parker, L. Bhuvimanian, E. Qasem, A.A. Mohammed, M. Zeeshan, K. Grugel, P. Carter, S. Ahmed, Short-term outcome of laparoscopic versus robotic ventral mesh rectopexy for fullthickness rectal prolapse. Is robotic superior?, Int. J. Colorectal Dis. 29(9) (2014) 1113-8.

[26] S.H. Emile, H. Elbanna, M. Youssef, W. Thabet, W. Omar, A. Elshobaky, T.M. Abd El-Hamed, M. Farid, Laparoscopic ventral mesh rectopexy vs Delorme's operation in management of complete rectal prolapse: a prospective randomized study, Colorectal Dis. 19(1) (2017) 50-57.

[27] A.H. Badrek-Al Amoudi, G.L. Greenslade, A.R. Dixon, How to deal with complications after laparoscopic ventral mesh rectopexy: lessons learnt from a tertiary referral centre, Colorectal Dis. 15(6) (2013) 707-12.

[28] M. Ahmad, P. Sileri, L. Franceschilli, M. Mercer-Jones, The role of biologics in pelvic floor surgery, Colorectal Dis. 14 Suppl 3 (2012) 19-23.

[29] D. Adeyemo, Mesh fistulation into the rectum after laparoscopic ventral mesh rectopexy, Int. J. Surg. Case Rep. 5(3) (2014) 152-4.

[30] M.L. Corman, A. Carriero, T. Hager, A. Herold, D.G. Jayne, P.A. Lehur, D. Lomanto, A. Longo, A.F. Mellgren, J. Nicholls, P.O. Nystrom, A.J. Senagore, A. Stuto, S.D. Wexner, Consensus conference on the stapled transanal rectal resection (STARR) for disordered defaecation, Colorectal Dis. 8(2) (2006) 98-101.

[31] G. Ribaric, A. D'Hoore, G. Schiffhorst, E. Hempel, T.R.S. Group, STARR with CONTOUR(R) TRANSTAR device for obstructed defecation syndrome: one-year real-world outcomes of the European TRANSTAR registry, Int. J. Colorectal Dis. 29(5) (2014) 611-22.

[32] A. Renzi, P. Talento, C. Giardiello, G. Angelone, D. Izzo, G. Di Sarno, Stapled trans-anal rectal resection (STARR) by a new dedicated device for the surgical treatment of obstructed defaecation syndrome caused by rectal intussusception and rectocele: early results of a multicenter prospective study, Int. J. Colorectal Dis. 23(10) (2008) 999-1005. 
[33] X.H. Ren, S.M. Yaseen, Y.L. Cao, W.C. Liu, S. Shrestha, Z. Ding, Y.H. Wu, K.Y. Zheng, Q. Qian, C.Q. Jiang, A transanal procedure using TST STARR Plus for the treatment of Obstructed Defecation Syndrome: 'A mid-term study', Int. J. Surg. 32 (2016) 58-64.

[34] A. Brescia, M. Gasparrini, U.M. Cosenza, G. Laracca, A. Milillo, A. Pancaldi, V. Vitale, F.S. Mari, Modified technique for performing STARR with Contour Transtar, Surgeon 11 Suppl 1 (2013) S19-22.

[35] J. Martellucci, P. Talento, A. Carriero, Early complications after stapled transanal rectal resection performed using the Contour(R) Transtar device, Colorectal Dis. 13(12) (2011) 1428-31.

[36] G. Dodi, R. Pietroletti, G. Milito, G. Binda, M. Pescatori, Bleeding, incontinence, pain and constipation after STARR transanal double stapling rectotomy for obstructed defecation, Tech. Coloproctol. 7(3) (2003) 148-53.

[37] M. Pescatori, G. Dodi, C. Salafia, A.P. Zbar, Rectovaginal fistula after double-stapled transanal rectotomy (STARR) for obstructed defaecation, Int. J. Colorectal Dis. 20(1) (2005) 83-5.

[38] M. Pescatori, G. Gagliardi, Postoperative complications after procedure for prolapsed hemorrhoids (PPH) and stapled transanal rectal resection (STARR) procedures, Tech. Coloproctol. 12(1) (2008) 7-19.

\section{Figures}




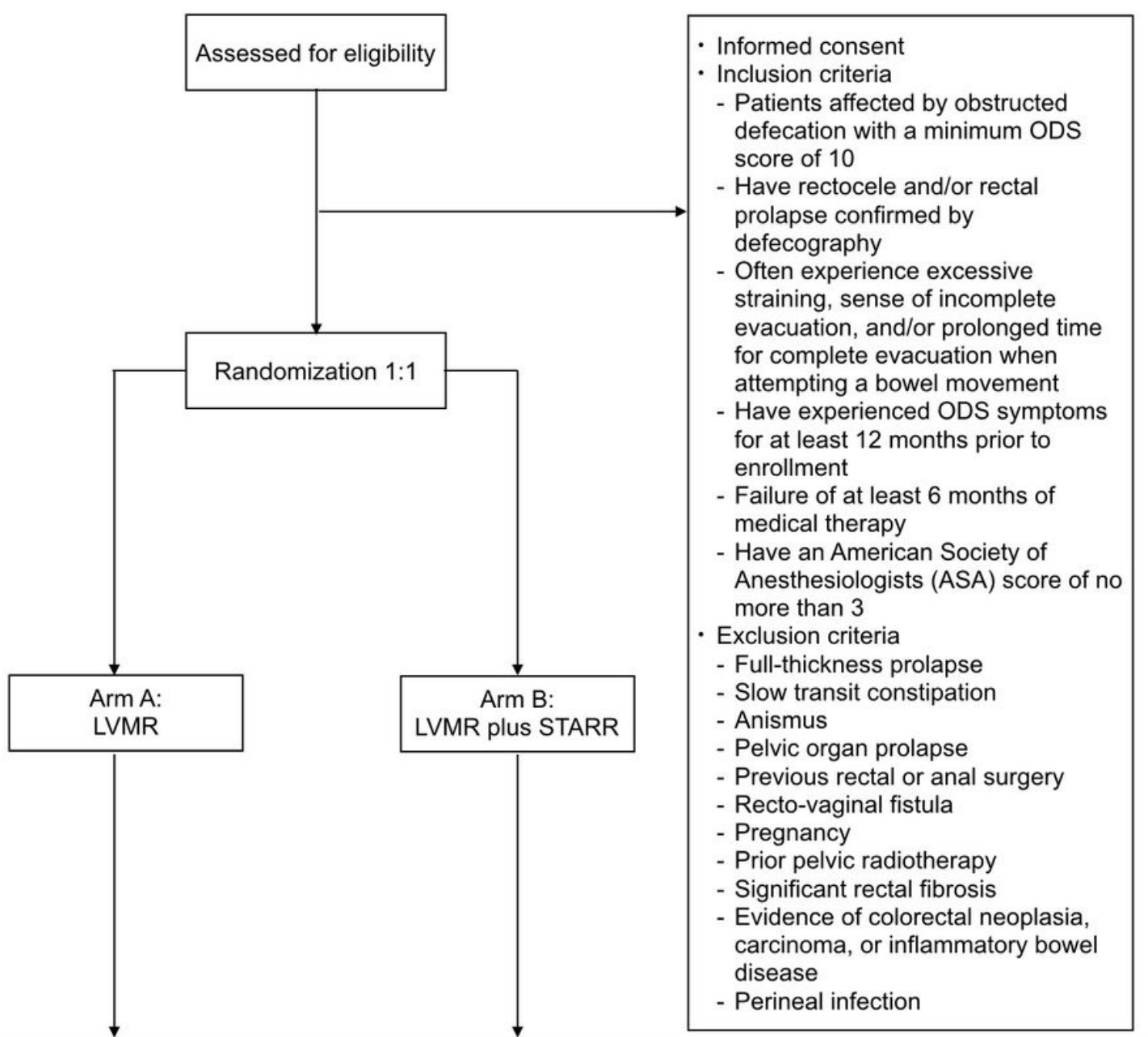

- Follow-up: at 1, 3, 6 and 12 months after the primary surgery

- The primary endpoint: the rate of complications started in the hospital and is followed up in the outpatient setting until 12 months after surgery.

- The secondary endpoints: the percentage of changes in the ODS score (ODS-S), the Patient Assessment of Constipation-Quality of Life score (PAC-QoL), the Health-Related Quality of Life SF-36 version 1, the Cleveland Clinic Fecal Incontinence Score (CCFI), the Fecal Incontinence Quality of Life Scale (FIQoL), between baseline and 1, 3, 6 and 12 months follow-up. Complications after surgery.

\section{Figure 1}

Diagram showing the flow of study design. 